\title{
APPLICATION OF MULTI-SOURCE REMOTE SENSING IMAGE IN YUNNAN PROVINCE GRASSLAND RESOURCES INVESTIGATION
}

\author{
Li Juanjuan ${ }^{1 *}$, Wen Guang ${ }^{1}$, Li Dongsheng ${ }^{2}$ \\ ${ }^{1}$ Yunnan Remote Sensing Center, 307352982@qq.com \\ ${ }^{1}$ Yunnan Remote Sensing Center, 2698334774@qq.com \\ ${ }^{2}$ Kunming Metallurgy College, 576268506@qq.com
}

Commission III, WG III/6

KEY WORDS: Remote Sensing Image, Grassland Resources, NDVI, Grassland Degradation, Vegetation Coverage, Ecological Protection

\begin{abstract}
:
Trough mastering background information of Yunnan province grassland resources utilization and ecological conditions to improves grassland elaborating management capacity, it carried out grassland resource investigation work by Yunnan province agriculture department in 2017. The traditional grassland resource investigation method is ground based investigation, which is time-consuming and inefficient, especially not suitable for large scale and hard-to-reach areas. While remote sensing is low cost, wide range and efficient, which can reflect grassland resources present situation objectively .It has become indispensable grassland monitoring technology and data sources and it has got more and more recognition and application in grassland resources monitoring research. This paper researches application of multi-source remote sensing image in Yunnan province grassland resources investigation .First of all, it extracts grassland resources thematic information and conducts field investigation through BJ-2 high space resolution image segmentation. Secondly, it classifies grassland types and evaluates grassland degradation degree through high resolution characteristics of Landsat 8 image. Thirdly, it obtained grass yield model and quality classification through high resolution and wide scanning width characteristics of MODIS images and sample investigate data. Finally, it performs grassland field qualitative analysis through UAV remote sensing image. According to project area implementation, it proves that multi-source remote sensing data can be applied to the grassland resources investigation in Yunnan province and it is indispensable method.
\end{abstract}

\section{THE INTRODUCTION}

In order to execute Party Central Committee and the State Council ecological civilization system reformation overall deployment to realize from quantitative control to entity control land resource management goal, it conducts county based grassland resource investigation by Yunnan province agriculture department and it finds out grassland resources utilization and ecological conditions in Yunnan province, which improves grassland elaborating management capability. It provides data support for deepening grassland ecological civilization system reform comprehensively.

Remote sensing technology and remote sensing data monitoring grassland, grassland resources has been getting more and more recognition and application both in domestic and abroad, which has become indispensable to grassland monitoring technology and data sources. The application of remote sensing technology in grassland science has been carried out since the early 1980s, and people have used the navigation and land resources satellite data to investigate, classify and map the grassland resources ( Wei Yaxing, Liu Chuang, Wang Liwen, 2007). However, using satellite data such as Landsat and MODIS to monitor and estimate grassland resources have only started in recent years. Due to the complex topography, large altitude difference, diversity climates, the investigation of Yunnan grassland resources project is time- short, heavy duty, demanding. This paper introduces the application of multi- source remote sensing image in grassland resources investigation in Yunnan province. Using Landsat 8 remote sensing image, it extracts investigation patch spectral characteristics, which is the determination base of grassland type. Using Zonal Statistics spatial analysis tool, the patch spectral characteristics are calculated, including the mean and variance of each band. The spatial correlation was established between sample patch and the remote sensing image. A set of spectral characteristics was extracted from each sample patch, and the spectral characteristics of each grassland type were distinguished. The spectral characteristics of each grassland type can be obtained by mean value of multiple samples spectral characteristics. After samples spectral characteristics statistical analysis, spectral characteristics of different grassland types can be formed, and the grassland properties can be determined according to the spectrum characteristic interval.

\section{GRASSLAND RESOURCES INVESTIGATION TECHNICAL SCHEME}

In this paper, through collecting and integrating land, surveying and mapping industry data, it obtains each stage remote sensing image. Then using each stage remote sensing image of grassland resources, it extracts thematic information, classifies grassland type, investigates field, assesses degradation degreed and estimates yield modeling. Finally using GIS and database information to establish grassland resources database, it forms report, statistics and thematic map which expresses all entities

\footnotetext{
* Corresponding author
} 


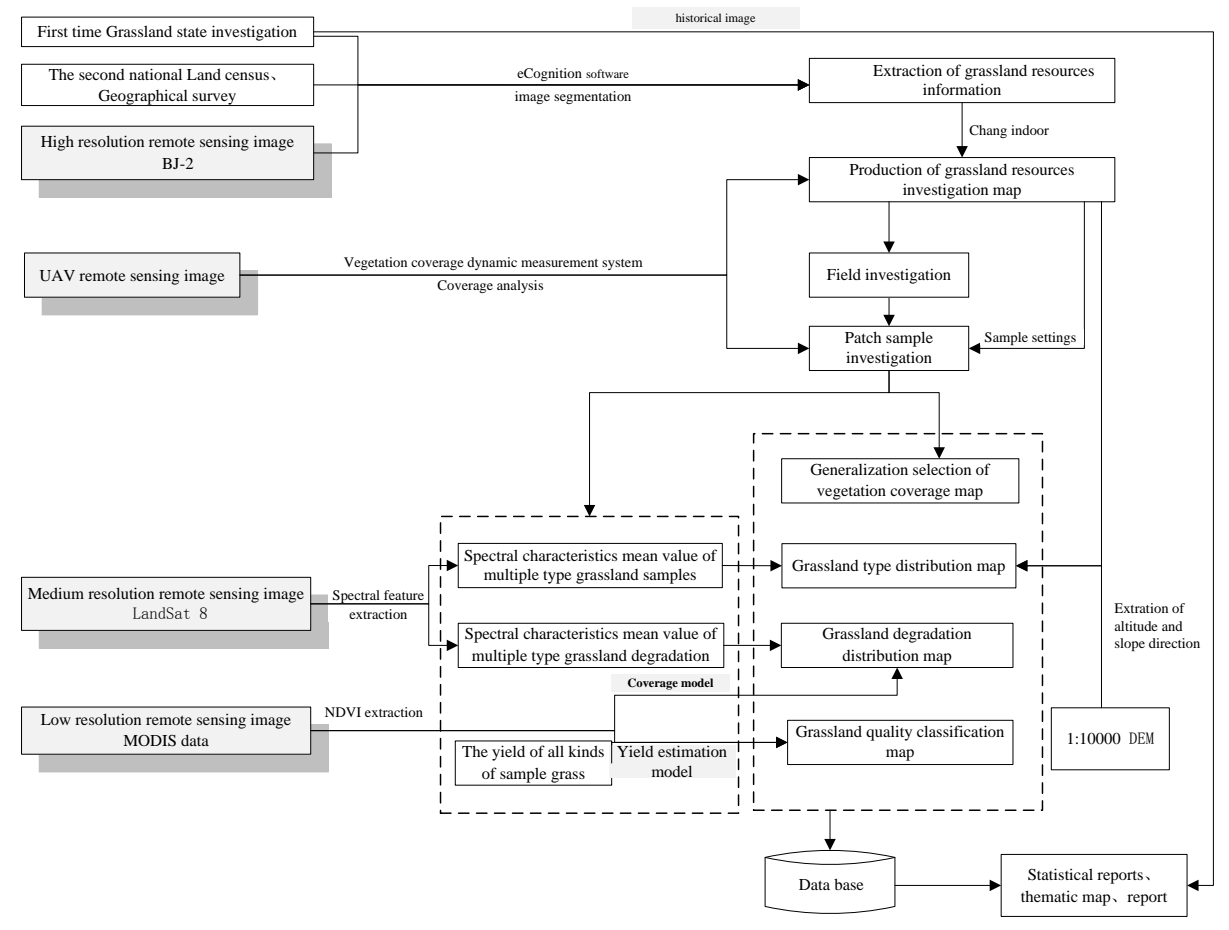

Figure 1. Grassland resources remote sensing image application flow chart

grassland resources distribution, quantity, quality, ecology and utilization objectively and standardly. The specific technical scheme is shown in Figure 1.

\section{APPLICATION OF MULTI-SOURCE REMOTE SENSING IMAGE}

\subsection{Extraction of grassland resources information}

The production of grassland resource investigation base map should use high-resolution remote sensing image that spatial resolution is higher than 10 meters and the acquisition time should be within 5 years. This paper uses high-resolution remote sensing image collected by BJ-2 (Beijing 2) that spatial resolution is $1 \mathrm{~m}$ and collecting time is 2016 . BJ-2 constellation is composed by three satellites which obtained 0.8 meters resolution panchromatic image and 3.2 meters resolution multispectral image(blue, green, red and near infrared spectrum) The image can clearly distinguish the cultivated land, trees and Scrub, grassland types. At first, through using eCognition software to take county as unit, this paper performs object orientation image segmentation under the second geographical state census results, which realizes grassland resources highresolution remote sensing image information extraction. Under the classification accuracy and extraction efficiency condition, it obtained grassland resources base map. Secondly, through artificial visual interpretation, the patch can be complemented and patch boundary can be corrected to form grassland resources thematic information. Finally, as to questioned patch, field investigation is conducted to form grassland resources base map.

\subsection{Grassland resources field investigation}

Grassland resources are vast, which is suitable to be investigated by satellite images (Graetz R D, 1987). The investigation contents include grassland type, grass yield, coverage, spatial distribution, and other environmental factors
( Purevdo rj T, Tateishi R, Ishiyama T, et al, 1998). In the process of project, it improves investigation grassland resource base map through patch supplement, patch boundary confirmation and attribute investigation referenced by BJ-2 high resolution image. Based on the BJ-2 remote sensing image, the spatial distribution and traffic conditions of various grassland were analyzed, and the sample layout and investigation were carried out to prepare for remote sensing yield estimation and quality classification.

\subsection{Generalization selection of vegetation coverage}

Before extraction of grassland resources, it is necessary to define the grassland type, which distinguishes grassland and grassland resources. One of the important criteria to distinguish between grassland and grassland resources is the coverage degree, which is distinguished by the coverage of trees or shrubs in the area. Secondly, it is necessary to determine the sample coverage accurately, so it obtains sample vegetation coverage and the whole county general vegetation coverage. And the general grassland utilization ratio of vegetation cover and crop straw is related to grassland resources, which are the second and third ecological development evaluation indexes respectively. Therefore, it is one of the most significant investigation work to obtain the comprehensive vegetation coverage of grassland efficiently and accurately. UAV (unmanned aerial vehicle) is small volume, light weight, low operation cost, high flexibility, higher resolution image and real-time transmission, which has become irreplaceable in the ecological environmental monitoring, agricultural production, natural disaster monitoring. This paper uses loaded infrared camera UAV, which connected to iPad to take real-time vegetation coverage image. Using vegetation coverage dynamic measurement system to analyze vegetation coverage, which can determine coverage type and coverage degreed quickly and objectively. 


\subsection{Evaluation of grassland properties}

Agricultural remote sensing made agricultural production and the research from the traditional stage entered to refinement, quantitative and mechanism stage (Xing Suli, Zhang Guanglu, 2003). The application of remote sensing image, especially in high resolution remote sensing image discriminating grassland type and the degradation degree (desertification, rocky desertification, salinization.) has been explored and conducted in domestic and abroad. The U.S. Landsat image has 30 meters resolution of about, and the ETM+ sensor has 6 to 7 visible bands and near-infrared bands that includes abundant spectral information, which can reflect soil, vegetation, water, rock information and so on, and even identify certain plant species. Therefore, the American Landsat image has great advantages in grassland type discrimination and degradation degree evaluation. Using Landsat 8 remote sensing image, it extracts investigation patch spectral characteristics, which is the determination base of grassland type. Using Zonal Statistics spatial analysis tool, the patch spectral characteristics are calculated, including the mean and variance of each band. The spatial correlation was established between sample patch and the remote sensing image. A set of spectral characteristics was extracted from each sample patch, and the spectral characteristics of each grassland type were distinguished. The spectral characteristics of each grassland type can be obtained by mean value of multiple samples spectral characteristics. After samples spectral characteristics statistical analysis, spectral characteristics of different grassland types can be formed, and the grassland properties can be determined according to the spectrum characteristic interval.

There is direct relationship between grassland type and geographical latitude, elevation and slope, and the grassland assignment is more accurate according to these factors. In this investigation, we extracted latitude, elevation and slope direction information from 1:10,000 high-precision digital elevation model ( DEM) produced by Yunnan remote sensing center to assign values to grassland types, which is the main way to discriminate the grassland properties. In the transition zone, it is complementary method through weight principle, spectral characteristics or field investigation. The evaluation results of grassland properties in Dongchuan county Kunming city are shown in Figure 2.

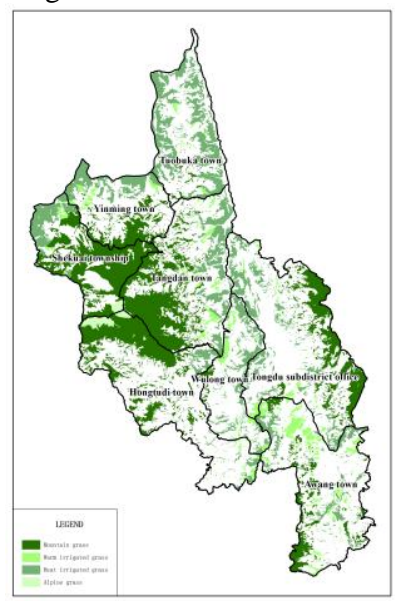

Figure 2. Grassland type map of Dongchuan County

\subsection{Evaluation of grassland degradation}

Grassland degradation refers to grassland productivity attenuation, excellent grass species is dried up, mixed grass is increased, and the grass coverage is atrophic under the influence of natural and human factors which caused underlying surface soil is bare ( Zha Yong, Gao Jay, Ni Shaoxiang, 2003). Grassland degraded remote sensing is mainly based on the remote sensing vegetation index change detection method ( Yaman o H, Chen J, Tamura M, 2003. TONG Chuan, XI Fengjiang, YANG Jingrong, et al,2003. Li Yunpeng, Na Risu, Liu Pengtao, et al, 2006. Li Suying, Li Xiaobing, Ying Ge, et al , 2007). The grassland degradation evaluation can be achieved through the three monitoring indexes, such as the coverage reduction rate, the grass yield reduction rate, and edible herbage reduction rate, which is realized according to the normalized vegetation index NDVI. In this paper, we adopt two indexes: coverage reduction rate and grass yield reduction rate. The implementation method of coverage reduction monitoring is: collecting 225th day of 2010 and 2017 MODIS NDVI data and using coverage model (coverage rate $=\frac{N D V I-N D V I_{\min }}{N D V I_{\max }-N D V I_{\min }} *$

100), it obtains 2010 and 2017 coverage distribution map. Then it obtains coverage reduction rate though 2017 coverage rate minus 2010 coverage rate, which assesses grassland degradation classification in Yunnan province. The implementation method of grass yield reduction monitoring is: collecting 225th day of 2010 and 2017 MODIS NDVI data and combing two year investigation sample data, yield estimation model, it obtains 2010 and 2017 grassland yield map. Then it obtains grass yield reduction rate though 2017 grassland yield minus 2010 grassland yield, which assesses grassland degradation. Choosing maximum value of the two assessment method as the grasslands degradation result.

The vegetation index can be used to estimate the biomass efficiently, at the beginning of grassland degradation, biomass may not decrease obviously. It may be only species change and biomass changes cannot be detected by vegetation index (Wang Huanjiong, Fan Wenjie, Cui Yaokui, et al, 2010). We obtained biomass changes by Landsat 8 images spectral characteristics. The similar attributes discrimination, it extracts spectral characteristics of each investigation sample patch. The spectral characteristics of degradation degree obtained by averaging value of multiple sample patch, which determines other grasslands patch degradation degree by grass spectral characteristic values Due to the uneven investigation quality sample and insufficient number of county-level samples, biomass is a supplementary method for the grassland degradation evaluation. The evaluation results of grassland degradation in Dongchuan county Kunming city are shown in Figure 3. 


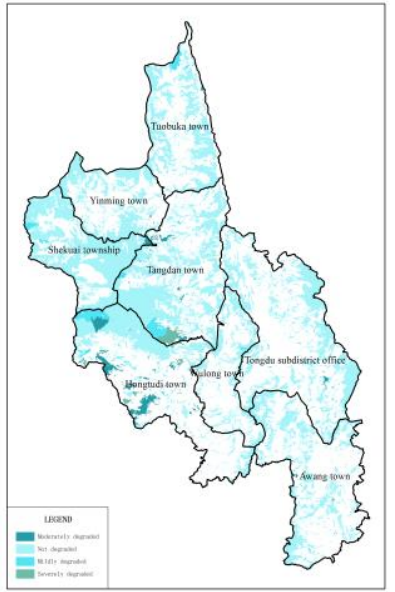

Figure 3. Grassland degradation distribution map of Dongchuan County

\subsection{Grassland quality classification}

The classification of grassland quality is one of the tasks of this investigation. The size of MODIS data is nearly $2000 \mathrm{~km}$ wide, and one orbit has unified radiation parameter. In MODIS data preprocessing, MRT tool can guarantee unified radiation correction, which can correct reflectivity level of each image to the same standard. It is difficult to achieve as to the medium and high resolution remote sensing image. Reflectivity is highly correlated with plant growth. In recent years, researchers have used remote sensing methods to estimate grassland biomass and in practice working, grassland biomass is replaced by NDVI (Zhang Pengli, Chen Jun, Cui Shujuan, et al, 2013). After analyzing the relationship between NDVI value and sample patch data of MOD13Q1_250m_16D_NDVI product $(250 \mathrm{~m}$ resolution, 2016 225th days), the results show that there is a significant correlation between them.

In this paper, the NDVI value of MOD13Q1_250m_16D_NDVI and grass dry weight were used to estimate the grassland yield classification model after the sample patch was converted as the standard hay. In ARCGIS, regional analysis was conducted to grassland patch and grassland yield, and grassland quality was graded according to grassland classification criteria. The results of the grassland quality classification in Dongchuan county Kunming city are shown in Figure 4.

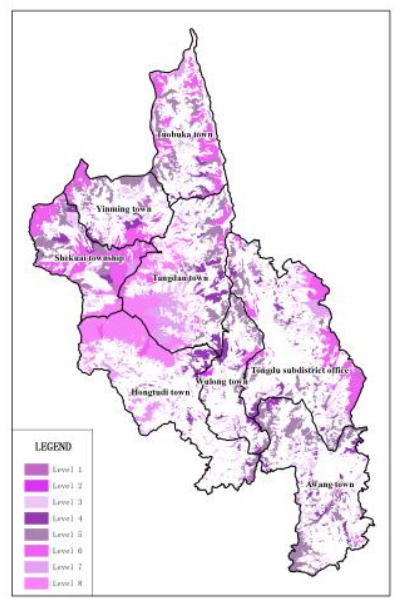

Figure 4. Grassland quality classification map of Dongchuan

\subsection{Grassland quality classification}

Remote sensing images can be used for dynamic grassland monitoring. It has been more than 30 years since the early 1980s. This 30 years, due to natural conditions change, the urban construction, overgrazing and grassland degradation, the quantity, quality and space distribution of grassland resources have taken place great changes in Yunnan province. Spatial heterogeneity is an important feature of grassland ecosystem ( Zhang Pengli, Chen Jun, Cui Shujuan, et al, 2013). Using variety of spatial scales and time image, it analyzed the change of grassland resources area space, scope, characteristic, reason and law in the past 30 years, which has far-reaching significance to grassland resource management, protection and rational utilization.

\section{CONCLUSION}

This paper summarizes the application of multi - source remote sensing image in Yunnan grassland resource investigation. Through county completed situation, it demonstrates that multisource remote sensing image applied to the grassland resources in Yunnan province is feasible and effective and it is very necessary. Remote sensing images have great advantages and broad prospects in grassland resource investigation, grassland monitoring and grassland occupation. Although we make full use of domestic and abroad public scale remote sensing image and technical strength in every process of grassland resources investigation, and is, the error caused by different spatial reference, different spatial scales, different time image fusion will affect the result of the investigation. The impact of each image source to investigation cannot be ignored, such as the mountainous shadow of $\mathrm{BJ}-2$ image, Landsat 8 image cloud cover area is large, and the resolution of MODIS image is too low. Only combed remote sensing technology and data with ground investigation technology, statistics, UAV GPS, database technology can guarantee grassland investigation effectively and accurately.

\section{ACKNOWLEDGEMENTS}

Paper is supported by Kunming metallurgy college fund (2017xjzk06). Thanks for multi-source remote sensing image supported by Yunnan remote sensing center 2017 basic national monitoring project and U.S. geological survey website.

\section{REFERENCES}

Wei Yaxing, Liu Chuang, Wang Liwen, 2007. Study on grassland monitoring based on remote sensing data. Science and Technology Consulting Herald, 27(020), pp. 23.

Graetz R D, 1987. Satellite remote sensing of Australian range lands. Remote Sensing of Environment, 23, pp. 313-331.

Purevdo rj T, Tateishi R, Ishiyama T, et al, 1998. Relationship between percent vegetation cover and vegetation indices. International Journal of Remote Sensing, 19(18), pp. 3519-3535.

Xing Suli, Zhang Guanglu, 2003. Application status quo and prospect of agriculture remote sensing in China. Transactions of the Chinese Society of Agricultural Engineering, 19(6), pp. 174-178. 
The International Archives of the Photogrammetry, Remote Sensing and Spatial Information Sciences, Volume XLII-3, 2018 ISPRS TC III Mid-term Symposium "Developments, Technologies and Applications in Remote Sensing", 7-10 May, Beijing, China

Zha Yong, Gao Jay, Ni Shaoxiang, 2003. Most recent progress of international research on remote sensing of grassland resources. Progress in Geography, 22(6), pp. 607-617.

Yaman o H, Chen J, Tamura M, 2003. Hyperspectral identification of grassland vegetation in Xilinhot, Inner Mongolia, China. International Journal of Remote Sensing, 24(15), pp. 3171-3178.

Tong Chuan, Xi Fengjiang, Yang Jingrong, et al, 2003. Remote sensing monitoring on degraded steppe and determination of reasonable grazing intensity for the restoration of steppe in middle reach of Xilin River Basin. Acta Prataculturae Sinica, 12(4), pp. 78-83.

Li Yunpeng, Na Risu, Liu Pengtao, et al, 2006. Remote sensing monitoring and climatic causes of Hulunbuir Grassland Degradation. Acta Agriculture Boreali - Sinica, 21(1), pp. 56-61.

Li Suying, Li Xiaobing, Ying Ge, et al , 2007. Vegetation indexes_biomass models for typical semi_arid steppe - a case study for Xilinhot in northern China. Chinese Journal of Plant Ecology, 31(1), pp. 23-31.

Wang Huanjiong, Fan Wenjie, Cui Yaokui, et al, 2010. Hyper spectral remote sensing monitoring of grassland degradation. Spectroscopy and Spectral Analysis, 30(10), pp. 2734-2738.

Zhang Pengli, Chen Jun, Cui Shujuan, et al, 2013. The community characteristics of different types of grassland under grazing prohibition condition. Acta Ecological Sinica, 33(2), pp. 0425-0434. 